\section{P1.60 ASSESSMENT OF E-TEST TO DETERMINE THE ANTIMICROBIAL SUSCEPTIBILITY OF NEISSERIA GONORRHOEAE}

Zhang Lijun, Peng Yi, Wang Feng. Shenzhen Centre For Chronic Disease Control, Shenzhen - China Popular Republic

\subsection{6/sextrans-2017-053264.165}

Introduction To evaluate the clinical value of E-test method to determine the antimicrobial susceptibility of Neisseria gonorrhoeae (N.gonorrhoeae) to five antibiotics.

Methods A total of 100 n.gonorrhoeae strains were collected. The agar-dilution method, E-test and K-B method were used to determine the minimum inhibitory concentration(MIC) of five antibiotics including ciprofloxacin, ceftriaxone, spectinomycin, penicillin and azithromycin. Assessment of agreement were made between the E-test and the reference agar dilution test. The comparisons include of discrepancy rate and correlation coefficients were made between the E-test and K-B method.

Results The MIC50,MIC90 and rang of MIC values were either identical or only differed by 0.5 to 1.5 dilution step. All antimicrobial agents had excellent essential agreement $(72 \%$ to $98 \%) \%$ ).Ciprofloxacin had the highest essential agreement (98\%),followed by azithromycin(92\%), while penicillin had the lowest essential agreement(72\%). In addition to the discrepancy rate with E-test for penicillin was higher than the K-B method, the other four antibiotics were just the opposite. The correlation coefficients between the E-test and the reference agar dilution test for other four antibiotics were significantly higher than the K-B method.

Conclusion The E-test method for ciprofloxacin and azithromycin can be used as an alternative method of agar dilution method used in clinical, but the E-test method for ceftriaxone and spectinomycin may lead the results to low. And the E-test method for penicillin is not suitable to used in clinical because of the low essential agreement and high minor discrepancy.

\section{LB1.61 PREVALENCE OF TRICHOMONAS VAGINALIS AMONG WOMEN ATTENDING A UNIVERSITY CLINIC IN PELOTAS, BRAZIL USING MICROSCOPY, CULTURE AND THE APTIMA TV ASSAY}

${ }^{1} \mathrm{MP}$ Bruni, ${ }^{2} \mathrm{MF}$ Silveira, ${ }^{2} \mathrm{D}$ Stauffert, ${ }^{1} \mathrm{CC}$ Santos, ${ }^{1} \mathrm{NAR}$ Farias, ${ }^{2} \mathrm{G}$ Bicca, ${ }^{3} \mathrm{D}$ Golparian, ${ }^{3} \mathrm{M}$ Unemo. ${ }^{1}$ Post Graduation Program in Parasitology, Biology Institute, Federal University of Pelotas (UFPel), Brazil, Brazil; ${ }^{2}$ Maternal and Child Health Department at the School of Medicine, Federal University of Pelotas (UFPel), Brazil, Brazil, ${ }^{3}$ Örebro University Hospital WHO Collaborating Centre for Gonorrhoea and other STIS, Sweden

\subsection{6/sextrans-2017-053264.166}

Introduction Trichomoniasis is a common sexually transmitted infection caused by the protozoan Trichomonasvaginalis (TV). In Brazil, trichomoniasis is not a reportable infection and the prevalence is underestimated, due to the poor sensitivity of routinely used diagnostic tests. Nucleic acid amplification tests (NAATs) are the most sensitive and specific tests available for TV detection, but TV NAATs are rarely used in Brazil. The objectives of this study were to investigate the prevalence of TV infection and compare the performance of the US FDAcleared APTIMA TV NAAT (Hologic, San Diego, USA) with wet mount microscopy, Gram stained microscopy and culture for diagnosis of TV infection in women in Pelotas, Brazil.
Methods From August 2015 to December 2016, 499 asymptomatic and symptomatic women attending the Gynaecology and Obstetrics Outpatient Clinic of the Maternal and Child Health Department at the School of Medicine, Federal University of Pelotas, Brazil were enrolled. Vaginal swabs were collected for examination with wet mount and Gram stained microscopy, culture, and Aptima TV assay. The Aptima TV assay was used as gold standard.

Results Participants' mean age was 37 years, the majority were white, had a steady sexual partner and low levels of education. The Aptima TV assay, culture, wet mount microscopy and Gram stained microscopy detected 4.2\% (21/499), $2.4 \%$ (12/499), $1.2 \%$ (6/499), and 0\% (0/499), respectively, of women as TV infected. The sensitivity and specificity of wet mount microscopy and culture were $28.57 \%$ and $100 \%$, and $57.14 \%$ and $99.79 \%$, respectively. The positive predictive value for wet mount microscopy was $100 \%$ and negative predictive value was $96.95 \%$ while for culture, these values were $92.30 \%$ and $98.14 \%$, respectively.

Conclusion Trichomoniasis was prevalent (4.2\%) in our population and the routine diagnostic test, wet mount microscopy, had very low (28.6\%) sensitivity compared to the Aptima TV NAAT. Improved diagnostic tests and enhanced testing of also asymptomatic patients are crucial to mitigate the transmission of TV infection.

\section{LB1.62 DETECTION OF SUBCLINICAL KIDNEY DISEASE IN HIV PATIENTS RECEIVING COMBINED ANTIRETROVIRAL THERAPY THROUGH NOVEL BIOMARKERS}

${ }^{1} \mathrm{~GB}$ Silva Junior, ${ }^{2} \mathrm{MG}$ Cavalcante, ${ }^{2} \mathrm{GC}$ Meneses, 'DS Sobral, ${ }^{1} \mathrm{AMC}$ Martins, ${ }^{1} \mathrm{JKB}$ Colares, ${ }^{1} \mathrm{DM}$ Lima, ${ }^{2} \mathrm{RJ}$ Pires Neto, ${ }^{2} \mathrm{EF}$ Daher. ${ }^{1}$ University of Fortaleza - UNIFOR, Brazil; ${ }^{2}$ Federal University of Ceara - UFC, Brazil

\subsection{6/sextrans-2017-053264.167}

Introduction Kidney disease is a well-known and frequent complication of HIV infection. The aim of this study is to investigate novel biomarkers of kidney disease in HIV patients.

Methods This is a cross-sectional study with HIV patients recruited in public health centres in Fortaleza city, Brazil, between January 2016 and March 2017. Three groups of HIV patients were included: I) those who have never received combined antiretroviral therapy (cART), II) those receiving cARTwithtenofovir/lamivudine/efavirenz or III) zidovudine/lamivudine/efavirenz. A group of 13 healthy subjects were the control group. The novel biomarkers investigated were: urinary and serum neutrophil gelatinase-associated lipocalin(uNGAL), urinary monocyte chemoattractant protein-1 (MCP-1) and urinary kidney injure molecule-1 (KIM-1), and the results were compared with the traditional biomarkers microalbuminuria and creatinine. Glomerular filtration rate (GFR) was estimated based on CKD-EPI equation.

Results A total of $66 \mathrm{HIV}$ patients were included, with mean age of $33 \pm 8$ years, and $77 \%$ were male. The majority of the patients $(63 \%)$ had undetectable viral load. Serum creatinine and GFR were similar in all groups. No HIV patient had GFR $<60 \mathrm{~mL} / \mathrm{min}$ and only two patients (3\%) had microalbuminuria $>30 \mathrm{mg} / \mathrm{g}-\mathrm{Cr}$ ).KIM-1 levels in patients using Tenofovir were higher than control group $(1.25 \pm 0.6$ vs 0.7 $\pm 0.2 \mathrm{ng} / \mathrm{mg}-\mathrm{Cr}, \quad \mathrm{p}<0.001)$.MCP-1 was significantly higher among HIV patients, and the highest values were found in those with no cART and high viral load. Urinary NGAL was 